\title{
JÜRGEN DITTMANN
}

\author{
Inhaltbezogene Grammatik
}


9. Inhaltbezogene Grammatik

1. Einleitung

2. Die sprachphilosophische Einordnung und der sprachtheoretische Standort der inhaltbezogenen Grammatik

3. Grammatiktheoretische Kategorien der inhaltbezogenen Grammatik

4. Ausgewählte morphosyntaktische Analysen

5. Satzmodelle als (auch) inhaltlich definierte Satztypen

6. Der Text als Gegenstand inhaltbezogener Sprachforschung

7. Abschließende Würdigung

8. Literatur

\section{Einleitung}

Die Beschäftigung mit der inhaltbezogenen Grammatik ist nicht nur von wissenschaftsgeschichtlichem Interesse. Vielmehr liegen ihrer Programmatik Fragestellungen zugrunde, die auch heute noch in Sprach- und Grammatiktheorie diskutiert werden. Vor allem geht es um das Problem der Beziehung zwischen Grammatik (Morphosyntax), Wortsemantik, Satzsemantik und Pragmatik natürlicher Sprachen. In jüngster Zeit sind dazu interessante Vorschläge von Vertretern der generativen Grammatik gemacht worden. So verficht Chomsky (1980, 59 ff) die Hypothese von der 'Modularität' des Sprachvermögens, womit er meint, man könne sinnvoll zwischen einer 'grammatischen Kompetenz' und anderen mentalen Fähigkeiten unterscheiden. Unter 'grammatischer Kompetenz' versteht er die Kenntnis von Form und Bedeutung natürlicher Sprachen (Morphosyntax, Lexik, Semantik) im Unterschied zur 'pragmatischen Kompetenz', die zusammen mit anderen konzeptuellen Systemen der Fähigkeit zugrundeliegt, die grammatische Kompetenz kommunikativ anzuwenden. Innerhalb des Grammatik-Moduls ist wiederum zwischen syntaktischen Regeln und der semantischen Interpretation syntaktischer Repräsentationen kategorial zu unterscheiden (vgl. u. a. Chomsky 1977, $36 \mathrm{ff}$; für eine revidierte Theorie der grammatischen Submodule vgl. Chomsky 1981, 135 ff). Auf Einzelheiten dieser Konzeption, z. B. die Rolle der Phonologie, und auch auf die mit der Hypothese von einer autonomen Syntax verbundenen Schwierigkeiten braucht hier nicht eingegangen $\mathrm{zu}$ werden, vielmehr ist für unseren Zusammenhang nur von Bedeutung, da $\beta$ die inhaltbezogene Grammatik in beiden genannten zentralen
Annahmen von diametral entgegengesetzten Auffassungen ausgeht: Die Sprachfähigkeit wird nicht als modular, sondern als mit anderen kognitiven Systemen interagierend angesehen, und zwischen Morphosyntax und Semantik wird nicht kategorial unterschieden. Die erste Grundannahme, die der modularen Konzeption der Sprachfähigkeit diametral entgegengesetzt ist, muß vor ihrem sprachphilosophischen Hintergrund gesehen werden: Es geht um die Frage nach dem Verhältnis von Sprache und Denken bzw. Sprache und Erkennen. Die sprachphilosophische und wissenschaftsgeschichtliche Einordnung der inhaltbezogenen Grammatik sowie ihre sprachtheoretischen Grundlagen werden im zweiten Abschnitt, die sprach- und grammatiktheoretischen Kategorien im dritten $\mathrm{Ab}$ schnitt behandelt. Die zweite Basisannahme, derzufolge zwischen Morphosyntax und Semantik nicht kategorial zu unterscheiden ist, erfordert in erster Linie die Definition von morphosyntaktischen Beschreibungskategorien in semantischen Termini, mithin gerade das, was durch die These von der Autonomie der Syntax ausgeschlossen wird (vgl. Leuninger 1979, 26f). Im vierten und fünften Abschnitt werden ausgewählte Beispiele morphosyntaktischer Analysen der inhaltbezogenen Grammatik diskutiert, in denen entsprechende Kategorien verwendet werden. Der sechste Abschnitt schließlich ist den einflußreichen Arbeiten von $\mathrm{H}$. Glinz gewidmet, deren Zuordnung zur inhaltbezogenen Grammatik allerdings nicht unstrittig ist.

\section{Die sprachphilosophische Einordnung und der sprachtheoretische Standort der inhaltbezogenen Grammatik}

Leo Weisgerber (1899-1985), der die Konzeption seiner inhaltbezogenen Grammatik aus der 'neuromantischen' Strömung der zwanziger Jahre heraus entwickelt (vgl. Gipper/Schmitter 1975, 546 ff), beruft sich für die Bestimmung des Verhältnisses von Sprache und Denken/Erkennen auf W. v. Humboldt (vgl. Weisgerber $1962 \mathrm{a}, 11 \mathrm{ff} u$. o..). Humboldt gebraucht mehrfach den Begriff einer sprachlich konstituierten 'Weltansicht': „Jede Sprache in jedem ihrer Zustände bildet das Ganze einer Weltansicht, indem sie Ausdruck für alle 
Vorstellungen enthält, welche die Nation sich von der Welt macht, und für alle Empfindungen, welche die Welt in ihr hervorbringt" (Humboldt $1906 \mathrm{ff}, \mathrm{Bd} . \mathrm{V}, 433$ ). Weisgerber interpretiert diese Auffassung im Sinne des sprachlichen Relativitätsprinzips, was zunächst plausibel erscheint. Allerdings sagt er nicht, daß sich nach Humboldt Weltansicht über die Lexik einer Sprache konstituiert (vgl. Heeschen 1977, 169), also nicht umstandslos auch mit der Grammatik der Einzelsprachen in Beziehung gebracht werden kann. Außerdem unterschlägt die einseitige Vereinnahmung Humboldts für das sprachliche Relativitätsprinzip seine rationalistische Grundposition, die ihn zum Postulat universaler Formprinzipien der Sprache führte (vgl. Humboldt 1906 ff, Bd. VII, 252; vgl. Dittmann 1980, 54). Während Humboldt also das Spannungsverhältnis von universellen und idiosynkratischen Zügen der Einzelsprachen erkennt, allerdings ohne es kategorial stringent zu erfassen, hebt Weisgerber einseitig auf letztere $\mathrm{ab}$. Er bringt darüber hinaus den Begriff 'Weltansicht' in Beziehung zu Humboldts Definition von Sprache als 'Energia', als einer wirkenden $\mathrm{Kraft}$, „die gemäß den Bedingungen und Möglichkeiten menschlichen Geistes dem Sein [...] in einer jeden Sprache zu einem Bewußt-Sein verhilft" (Weisgerber $1962 \mathrm{a}, 33$ ). Mit dieser Umdeutung von Weltansicht in 'WeltBILD' als „Zugriff" der 'Muttersprache' auf Wirklichkeit kommt Weisgerber einer deterministischen Verschärfung des Relativitätsprinzips sehr nah, die keinesfalls von Humboldt her zu rechtfertigen ist (vgl. Heeschen 1977, $170 \mathrm{ff}$ ). In seinem bekanntesten Werk, den „Grundzügen der inhaltbezogenen Grammatik", formuliert Weisgerber (1962 a, 33) programmatisch, es gehe ihm ,letztlich um das Weltbild der deutschen Sprache“. Damit ist gemeint, daß „Grammatik im weitesten Sinne“ als „Verfahren des Bewußtwerdens der ganzen Sprache, mit Einschluß des Wortschatzes" zu verstehen sei (ebd., 28). Der traditionellen Sprachwissenschaft wirft er vor, den Inhalt der Sprache als Anhängsel der sprachlichen Lautmittel aufgefaßt und außerhalb der Sprache (im Denken oder in der Außenwelt) angesiedelt zu haben (vgl. ebd., $29 \mathrm{f}$ ). Für ihn rücken dagegen in der ,grammatischen Beschreibung einer sprachlichen Weltansicht" die sprachlichen Inhalte ins Zentrum des Interesses, die als „geistige Zwischenwelt“ zwischen der sog. Außenwelt und dem menschlichen Bewußtsein vermitteln (vgl. ebd., 38 ff).
Im Bereich der Lexik läßt sich, was Weisgerber damit meint, mit einem Wort wie Unkraut illustrieren: In gewisser Weise gibt es in der Natur kein Unkraut, sondern der Mensch stempelt bestimmte Pflanzen als Unkräuter $a b$, sie ,[werden] erst in einer geistigen Zwischenwelt möglich" (ebd., 57). Befangen in seinem rigorosen Sprachidealismus, geht Weisgerber mit keinem Wort darauf ein, daß sich eine Kategorisierung in Kräuter und Unkräuter letztlich der Praxis des menschlichen Umgangs mit der Natur verdankt. - Anspruch der inhaltbezogenen Grammatik ist es aber darüber hinaus, die grundlegende sprachtheoretische Perspektive auch in der Morphosyntax, bei der Beschreibung der „Mittel der Redefügung“ (vgl. ebd. 1962 a, 83) durchzuhalten. Allerdings sieht Weisgerber selbst hier ein grundsätzliches Problem: Seine Zeichentheorie (vgl. ebd., $78 \mathrm{ff}$ ) geht von der These aus, 'Sprachmittel' seien als Ganzheiten aus Lautform und Inhalt anzusehen, eine These, als deren Vertreter Weisgerber auch Saussure anführt. Angewendet auf die Morphosyntax, müßte diese These eine „möglichst enge Beziehung zwischen Einheiten formaler Art und syntaktischen Inhalten" (ebd., 83) erwarten lassen, die jedoch, wie Weisgerber sehr wohl sieht, so nicht existiert. Als Beispiel führt er die Kategorie 'Genitiv' an, der formal eine „Mannigfaltigkeit" entspreche (des Mannes, der Frau usw.). Daraus folgt die Notwendigkeit, grammatische Inhalte losgelöst von Beschränkungen durch formale Gliederungen zu beschreiben.

\section{Grammatiktheoretische Kategorien der inhaltbezogenen Grammatik}

Weisgerber fordert als Konsequenz seiner Sprachtheorie eine ,ganzheitliche Sprachforschung" (1962a, 22) in der jedes Phänomen (z. B. der Kasus Akkusativ oder das Passiv) - modern gesprochen - einer morphologischen, syntaktischen, semantischen, pragmatischen und (de facto, wenngleich programmatisch weniger deutlich) einer sprachhistorischen Betrachtung unterzogen wird. Hierzu unterscheidet Weisgerber ,vier Stufen in der Erforschung der Sprachen": Die erste Stufe ist die zunächst „lautbezogen“ (vgl. Weisgerber 1953, 23 f), später (vgl. Weisgerber $1963 \mathrm{c}$, $15 \mathrm{f})$ zutreffender „gestaltbezogen“ genannte Betrachtung, die der traditionellen Grammatik entsprechen soll. Sie erhebt mit ausdrucksseitig orientierten Verfahren den sprachlichen 
Bestand. Im Bereich der Morphosyntax gehört dazu das Aufstellen von Flexionsparadigmen und die Definition von morphosyntaktischen Kategorien, wie 'Nomen', 'Verb', 'Kasus', 'Tempus' usw. (vgl. Weisgerber 1962 a, $297 \mathrm{ff}$ ). Die zweite Stufe ist die „inhaltbezogene Betrachtung" ( $\mathrm{da} \beta$ 'inhaltbezogen' zugleich als Oberbegriff für alle vier Stufen fungiert, ist ein terminologischer Mißgriff). Sie wird in die „Lehre von den Wortinhalten“ (Wortsemantik/Lexikographie) und die „Lehre vom inhaltlichen Aufbau der Mittel der Redefügung" (semantisch orientierte Morphosyntax) unterteilt (vgl. ebd., 138). Da im vorliegenden Zusammenhang die wortsemantische Anwendung (in deren Zentrum die Wortfeld-Theorie steht; vgl. Geckeler 1971, $84 \mathrm{ff}$ ) nicht weiter interessiert, sei ein Beispiel für ein inhaltbezogenes Raisonnement aus dem Bereich der Morphologie angeführt: Weisgerber (vgl. $1962 \mathrm{a}, 324 \mathrm{ff}$ ) kritisiert die Gleichsetzung von Tempuskategorien (wie 'Präsens' oder 'Futur') mit Zeitbezügen (wie 'Gegenwart' oder 'Zukunft') und hält dem u. a. die wahrscheinliche Interpretation von Ich gehe nach Hause. i. S. des Zukunftsbezuges, die eindeutig zukunftsbezogene Interpretation von Ich gehe morgen in die Stadt. und die wahrscheinlich vergangenheitsbezogene von Ich finde da gerade eine wichtige Nachricht. entgegen: Dem einen formalen Paradigma entspricht eine inhaltliche Mannigfaltigkeit. - Die dritte Stufe heißt ,leistungbezogene" Betrachtung, und sie, nicht etwa die inhaltbezogene Betrachtung, stellt das Kernstück der Sprachbetrachtung dar (vgl. Weisgerber 1963c, 111). Von den sprachtheoretischen Prämissen her gedacht ist das plausibel, denn die leistungbezogene Betrachtung zielt auf das spezifisch energetische (von 'Energeia') Moment im Sprachlichen, auf die Analyse des Prozesses der „sprachlichen Anverwandlung“, des „Wortens der Welt" in der Muttersprache (vgl. ebd., 94; 96). Energetisches Pendant zum statischen sprachlichen Inhalt ist auf dieser Stufe der „sprachliche Zugriff" (vgl. ebd., 96). Es geht, mit Gipper $(1974,144)$ formuliert, ,um das Bewußtmachen der spezifischen geistigen Verfahrensweisen, mit denen eine Sprache ihren Sprechern einen unverwechselbaren Zugang zur Welt eröffnet". (Man beachte hier die Verwendung von Sprache als Nomen actionis, eine Redeweise, auf die zurückzukommen sein wird!) In der Anwendung ist die Abgrenzung zur inhaltbezogenen Betrachtung nicht immer deutlich. So führt Weisgerber $(1964,39)$ am
Beispiel der Diminutivbildungen aus, Suffixe wie -lein und -chen würden substantivische Begriffe in die "Sehweise des Verkleinerten“ bringen, eine Sehweise, die Bestandstück des muttersprachlichen Weltbildes sei. Man könne sich fragen, ob es in der 'Wirklichkeit' überhaupt Häuschen und Kindlein gebe, oder ob darin nicht ein gut Stück menschlichen Urteils stecke. Letztere Frage ist freilich allenfalls als rhetorische zulässig: Selbstverständlich ist es menschlicher Maßstab, der hinter der Unterscheidung von Haus und Häuschen steht. Nicht klar wird aber auch, was denn, wenn nicht die „Sehweise des Verkleinerten", den 'Inhalt' der Diminutiv-Suffixe ausmachen soll. Eine nicht-triviale Feststellung liegt hingegen vor, wenn Weisgerber in bezug auf die Diminutiv-Bildung von einer „dauernden Bereitstellung dieser Möglichkeit als Leistung der Muttersprache" in dem Sinne spricht, daß die deutsche Sprache keine entsprechenden Vergrößerungsformen bereitstellt. Der rationale Kern der mehr metaphorischen als präzisen Ausführungen zur leistungbezogenen Betrachtung liegt deshalb zum einen im Hinweis auf die Relevanz einzelsprachlicher Unterschiede in der Gliederung von Bedeutungsfeldern (Wortsemantik) und morphosyntaktischer Ausdrucksmittel, zum anderen im Insistieren auf der Relevanz des Spracherwerbs für die kognitive Entwicklung des Kindes. Letzteren Komplex hat Gipper mehrfach behandelt, wobei seine Basishypothese in Aussagen wie der folgenden deutlich wird: „Das Kind erobert seine Welt am Leitfaden sprachlicher Gliederungen, die es in unzähligen Kommunikationsakten mit den Erwachsenen erprobt und aneignet" (Gipper 1974, 139). - Die vierte und letzte Stufe ist die der „wirkungbezogenen“ Betrachtung, auf der, laut Gipper (ebd., 144), die pragmatische Seite der Sprachverwendung mit einbezogen wird. Weisgerber (1962 a, 24) begründet die Notwendigkeit einer wirkungbezogenen Sprachbetrachtung mit dem Hinweis darauf, Sprache sei nicht Selbstzweck, sondern gehe als mitgestaltende Kraft in die Handlungen der Menschen ein. Deshalb gehörten auch die sprachlichen Wirkungen, die sich dabei ergäben, zu den notwendigen $\mathrm{Ge}-$ genständen der Sprachwissenschaft. - Wissenschaftsgeschichtlich gesehen ist die wirkungbezogene Sprachbetrachtung der umstrittenste Teil der inhaltbezogenen Grammatik. Das liegt weniger daran, daß die inhaltbezogene Grammatik sich, so Helbig $(1974,138)$ durch die Thematisierung von 
Sprache als „Teilkraft im Aufbau der Gesamtkultur“ (ebd., 24), also durch einen ,geistesgeschichtlichen Ausbau“ vom „sprachlichen Objekt" entfernt hat. Diese Entwicklung hat sich ja in anderen Richtungen der Sprachwissenschaft spätestens durch die 'pragmatische Wende' Anfang der siebziger Jahre ebenfalls vollzogen, wenngleich mit anderen theoretischen Bezügen (vgl. Dittmann 1980, 66). Problematisch ist vielmehr die einseitige Perspektivierung dieser 'pragmatischen' Analysen: Nach weithin akzeptiertem Verständnis hat es die Pragmatik als semiotische Teildisziplin, grob gesprochen, mit dem Verhältnis von Zeichen, Referenzbereichen, Zeichenbenutzern und Gebrauchskontexten zu tun, wobei ein Schwerpunkt auf der Frage liegt, wie - mit welcher Absicht und welcher Wirkung (!) - Zeichen gebraucht werden. In linguistischer Sicht ist also die Ebene der Sprachverwendung betroffen. Weisgerber hingegen interessiert sich letztlich für die Frage nach Wirkungen sprachlicher Ausdrucksmittel (Ebene des Sprachsystems) in der bzw. auf die Sprachgemeinschaft. Handlungsträger in diesem Wirkungszusammenhang sind nicht die sprechenden Individuen, sondern, metaphorisch, die sprachlichen Ausdrucksmittel. Deshalb ist für Weisgerber $(1963 \mathrm{c}, 172)$ das „Handeln der Sprachgemeinschaft" eine Form der Aktivität, die ,durch die Richtung der geltenden Sprachzugriffe gelenkt wird“" und deshalb nicht als Summe von Einzelaktivitäten begriffen werden kann. Wohlgemerkt: auch Weisgerber wählt in seinen sprachtheoretischen Exkursen Ausdrucksweisen wie die, der Mensch ,verfüge“ über Sprache, und er spricht explizit von den „Wechselbeziehungen zwischen Sprache und Leben“" (Weisgerber 1957/58, 199, 202). Aber sowohl programmatisch als auch in der Anwendung betont die energetische Sprachbetrachtung die aktive Rolle der Sprache, den Aspekt, daß Sprache (im Sinne der Beherrschung der Muttersprache, nicht im Sinne von 'Rede' bzw. 'sprachliche Kommunikation' aufgefaßt) ,an der Bewältigung der Aufgaben des Lebens mitbeteiligt ist" (ebd., 202), eine Auffassung, die sich grammatisch in einer Redeweise niederschlägt, in der Sprache als Nomen actionis auftritt (vgl. oben in diesem Abschnitt zu Gipper 1974, 144). - Problematisch an dieser Hypostasierung von Sprache als wirkende Kraft ist die Verwischung der Trennlinie zwischen Sprache und ihrem Gebrauch in Äußerungen bzw. Texten. Das wird anhand der wirkungbezogenen Betrachtung des Adj. deutsch besonders anschaulich (vgl. Weisgerber 1963 c, $173 \mathrm{ff}$ ): Gegen Weisgerbers Darstellung muß vehement eingewendet werden, daß nicht die Existenz des ahd. diutisk als solche Richtung des politischen Wollens und Handelns der Menschen in der östlichen Reichshälfte im 9. Jahrhundert „,entscheidend mitbestimmt", sondern der Gebrauch, den Politiker von diesem Wort gemacht haben. In diesem konstituiert und perpetuiert sich erst die spezifische Bedeutung von diutisk als politischem Begriff. Die Umkehrung dieser Perspektive führt zum Teil, so bei Tschirch (vgl. 1954, 86), zu absurden Konsequenzen (vgl. die Kritik bei Helbig 1974, 142 ff; Dittmann 1980, 72 ff). - Die Anwendung der wirkungbezogenen Betrachtung auf die Morphosyntax erläutert Weisgerber (1963a, $272 \mathrm{f}$ ) unter anderem am „Wortstand“ (Wortstände sind inhaltlich definierte Gruppen von Ableitungen in der Wortbildungslehre) der „Zugänglichkeitsadjektive“. Formal unterscheidet er hier drei Ableitungstypen: den auf -bar (hörbar), den auf -lich (löslich) und den auf -sam (biegsam). Gemeinsam ist ihnen nach Weisgerber inhaltlich, daß sie Größen kennzeichnen, die der Aktion des Verbs unterstellt werden, jedoch unterscheidet sich der Inhalt in Nuancen: Beim dritten Typ wird diese Größe ,als einer solchen Behandlung entgegenkommend“, beim zweiten als „mit Erfolg zugänglich“, beim ersten als „unterschiedslos unterstellbar" gekennzeichnet. Die leistungbezogene Perspektive drückt sich in der Feststellung aus, da $B$,dieser Wortstand eine Beurteilung der Objekte auf ihre Zugänglichkeit für Handlungen nicht nur erlaubt, sondern daß er diese veranlaßt, herbeiführt" Sprachgemeinschaft wird „diese Richtung des Beurteilens [...] aufgedrängt". Die wirkungbezogene Betrachtung arbeitet zusätzlich heraus, daß das sprachhistorisch zu konstatierende Zunehmen der Bildungen auf -bar in der Sprachgemeinschaft der Anstoß ist, den „Gesichtspunkt der unterschiedslosen $\mathrm{Zu}$ gänglichkeit allen möglichen Objekten gegenüber anzuwenden und diese in der Weiterwirkung auf ihre Zugänglichkeit und Verwendbarkeit zu prüfen" (ebd., 273). Dieser Wortstand drängt also, so Weisgerber, den Menschen eine Perspektive im Denken auf und leitet sie an, diese Perspektive auszuweiten, wobei diese Einwirkung gerade dadurch besonders wirksam ist, daß sie normalerweise unbewußt bleibt. - Anhand des Beispiels der Zugänglichkeitsadjektive läßt sich ein typisches Problem der inhaltbezogenen gramma- 
tischen Beschreibungsbegriffe gut verdeutlichen: Die Kategorie 'mit Erfolg zugänglich' (-lich) ist offenbar aus Beispielen wie erklärlich gewonnen, die sozusagen einen positiven Bedeutungsaspekt haben. Wie diese Beschreibung z. B. auf unerklärlich zutreffen soll, ist nicht nachvollziehbar. Tatsächlich müßte man sich für diese Ableitungen wohl auf einen neutralen Terminus wie 'Dispositionsbegriffe' einigen.

\section{Ausgewählte morphosyntaktische Analysen}

\section{1. Über den Akkusativ}

Exemplarisch sei zunächst die bekannteste Studie zur inhaltbezogenen Morphosyntax aus dem Kreis der inhaltbezogenen Grammatik vorgestellt, Weisgerbers „Der Mensch im Akkusativ". Die zugrundeliegende Hypothese lautet: „Es scheint, daß in der heutigen deutschen Sprache einer Verfahrensweise verstärkt Gewicht zukommt, die Personen, die in ein Geschehen einbezogen sind, in die sprachliche Rolle des Akkusativs bringt" (Weisgerber 1957/58, 193). Hinter dieser unmittelbaren Verkoppelung einer morphosyntaktischen Form und einer bestimmten Sehweise der Mitglieder der Sprachgemeinschaft steht, sprachtheoretisch, letztlich die Determinismusthese, und programmatisch zielt die Hypothese auf die leistung- und wirkungbezogene Betrachtung. - Entsprechend den vier Stufen der ganzheitlichen Sprachbetrachtung setzt auch diese Untersuchung mit der lautbezogenen Betrachtung ein, nämlich mit dem Aufzeigen der Wortbildungsmöglichkeiten transitiver Verben mit „personalen Akkusativobjekten“. Als Hauptquelle für Neubildungen macht Weisgerber die be-Ableitungen (frei/befreien, ruhig/beruhigen usw.) aus. Den Übergang zur inhaltbezogenen Betrachtung bildet erstens die Definition einer inhaltlichen Kennzeichnung für solche Wortbildungstypen. Im Fall der be-Ableitungen handelt es sich nach Weisgerber um Verben, bei denen „Ableitungen aus Substantiven auf das Ausstatten mit dem im Substantiv Gemeinten hinweisen“" (1957/58, 197). Zweitens versucht er den Nachweis zu führen, daß auch andere formale Bildungsmöglichkeiten von Verben diesen bzw. zumindest einen ähnlichen inhaltlichen Effekt haben, so die Suffigierung mit -igen (benachrichtigen $=$ mit Nachricht versehen). Zusammengefaßt machen diese Verben den Wortstand der 'Ornative' (von lat. ornare $=$ versehen, ausstatten $)$ aus (vgl. ebd., 198). Am Ende der inhaltbezogenen Betrachtung zeichnen sich bereits ,die geistigen $\mathrm{Zu}$ sammenhänge $a b$, in denen der Anlaß zur 'Akkusativierung' gegeben ist; es sind bestimmte Sehweisen, die in der Sprache ausgebildet sind und die als Möglichkeiten oder noch besser als Anleitung für den Sprachgebrauch bereitstehen“"(ebd., 199). - Damit ist die erste Stufe der energetischen, die leistungbezogene Betrachtung erreicht. Was zunächst als Inhalt aufzuzeigen war, wird nun als „Akt der sprachlichen Anverwandlung der Welt" in der Muttersprache beschrieben, hier genauer: als "geistiger Zugriff“ in Gestalt des Wortstandes der Ornative. Die Ausgangsfrage ist deshalb: Was geschieht, wenn im sprachlichen Zugriff der Mensch in die Rolle des Akkusativobjektes gebracht wird?" (ebd., 200). Und sie wird mittels eines interpretativen Verfahrens beantwortet, das von einem Vergleich zwischen dem „Menschen als Dativobjekt" und dem „Menschen als Akkusativobjekt" ausgeht. Das nach Weisgerber im 19. Jahrhundert vorherrschende einem rufen unterscheide sich vom heute geläufigeren einen rufen dadurch, daß im ersten Fall der Ruf sich an den in seiner Entscheidung frei gesehenen Menschen richte, während im anderen Fall in dem Anruf bereits der Anspruch auf das Willfahren des Angerufenen gedanklich vorweggenommen werde. Der Mensch im Dativobjekt erscheine als die Stelle, von der her das ganze Geschehen seinen Sinn gewinne, im Akkusativobjekt dagegen sei der Mensch Schauplatz eines gedanklichen Eingriffs, also Objekt im vollen Sinne (ebd., 200). $\mathrm{Zu}$ einem telefonieren vs. einen anrufen schreibt Weisgerber gar: „Die Belästigung des Angerufenen wird mir durch den Akkusativ viel leichter gemacht" (ebd., 201). Spätestens hier wird ein gravierendes methodisches Manko der inhaltbezogenen Grammatik deutlich: Da es sich bei einem vs. einen anrufen nicht nur um eine sprachhistorische Erscheinung, sondern auch um dialektale Varianten handelt (alemannisch z. B. steht ausschließlich der Dativ), müßte Weisgerber zunächst klarstellen, für welche Varietät(en) er seine Befunde erhebt (vgl. Steger 1964, 126f). In den am konkreten Beispiel, der Akkusativierung, aufgezeigten ,geistigen Bahnen“ verläuft mithin für Weisgerber das Denken der Sprachgemeinschaft, die ,übliche Art, mit den Erscheinungen geistig umzugehen", hier: daß „die Menschen immer und immer wieder in die Rolle des Akkusativobjektes" gebracht 
werden (Weisgerber 1957/58, $201 \mathrm{f}$ ). - Mit dieser leistungbezogenen Betrachtung ist die Analyse weitgehend abgeschlossen. Was die wirkungbezogene Betrachtung noch beiträgt, ist ein Versuch der geistesgeschichtlich-ideologiekritischen Einordnung der 'Akkusativierung'. Weisgerber sieht sie im Kontext bestimmter „Lebensverhältnisse“, wie „Betreuungswesen" (wegen betreuen, berenten usw.), „statistisches Erfassen“ und „Mißbrauch der Macht" (Menschen verplanen usw.; ebd., 202f). Dabei thematisiert er zwar das Problem der Wirkungsrichtung: „Wie weit sind die sprachlichen Akkusativierungen Parallelen, Folgen [!], Ursachen dieser Erscheinungen des Lebens?" Aber von seiner sprachtheoretischen Ausgangsposition her ist es selbstverständlich, daß er letztlich auf die deterministische Perspektive abhebt: Die Sprache habe nicht lediglich die Rolle eines Spiegels gehabt, sondern sie habe ,auch von sich aus akkusativierende Anstöße hinzugebracht, die sich dann entsprechend in der Gestaltung dieser Lebensgebiete auswirkten“. Da er immerhin konzediert, daß das Leben auch „,von sich aus" die Bewältigung solcher Aufgaben herbeigeführt und insofern kausal an der sprachlichen Erfassung beteiligt gewesen sein könnte, kann man Weisgerbers These wohl dahingehend zusammenfassen, die modernen Lebensverhältnisse in der Massengesellschaft begünstigten einerseits die Bildung personaler Akkusativobjekte, andererseits wirke die sprachliche Möglichkeit ihrer Bildung in die Gesellschaft hinein, zumindest in die „Ausgestaltung" solcher Lebensgebiete wie Verwaltung, Militär und Wirtschaft (vgl. ebd., 204). - Bei genauerem Hinsehen wird zunächst klar, daß Weisgerber die gestaltbezogene Betrachtung nicht sorgfältig genug durchgeführt hat. Dadurch sind ihm innersprachliche Gründe, die die Bildung von Akkusativierungen begünstigen, entgangen (vgl. Kolb 1960, 170 ff): U. a. liegt ein Vorzug der $b e$-Verben darin, daß sie die Nennung der „akkusativierten“) Person verlangen, die der präpositional damit verknüpften Sache aber freistellen (er beschenkt seine Kinder, fakultativ: mit Spielzeug), während bei den Grundverben die Angabe des Sachobjekts obligatorisch ist (er schenkt seinen Kindern Spielzeug). Andererseits ist bei diesen die Angabe des personalen Objekts fakultativ (ich schenke Spielzeug). Diese und weitere immanent sprachliche Erscheinungen beeinflussen die 'Wahl' zwischen Grundverb und be-Erweiterung, ohne daß sich für die Sprecher/innen damit ,'Verschiebungen in der sprachlichen Einschätzung von Menschen und Sachen' (Weisgerber) verbinden“" (ebd., 172). Darüber hinaus zeigt sich, daß die Akkusativierung mittels be-in der deutschen Sprachgeschichte seit Jahrhunderten eine große Rolle spielt. So findet sich nach Kolb im Wortschatz der Rechtssprache vor 1700 eine erstaunlich hohe Zahl dieser Verben, die heute zum Teil gar nicht mehr existieren. Damit ist folgendes gezeigt: Erstens begünstigen innersprachliche Faktoren die Produktivität dieser Ableitungen und können sie somit zumindest ein Stück weit erklären; zweitens kann die 'Akkusativierung' zumindest nicht allein auf das Konto der modernen gesellschaftlichen Verhältnisse gebucht werden (vgl. auch Henzen 1959, $207 \mathrm{f}, \mathrm{zu}$ helfen); drittens spielt sie in fachsprachlichen Bereichen eine besondere Rolle, die ohnehin durch Ökonomie- und Präzisionsstreben gekennzeichnet sind (vgl. Kolb 1960, 176 f). Kolb kommt deshalb zu dem Schluß, die Akkusativierungen seien nur insoweit bezeichnend für die geistige Haltung des modernen Massenzeitalters, wie diese Bereiche in der modernen Gesellschaft wirksam seien, als solche seien sie jedoch „kein Zeichen der Zeit. Kulturpessimismus und Zeitkritik finden an ihnen einen untauglichen Gegenstand. Der Akkusativ ist weder inhuman noch human, sondern eine grammatische Form, die von human und inhuman Gesinnten gebraucht werden kann" (ebd., 177; vgl. Henzen 1959, 207). - Dieser Kritik ist, was das Aufweisen innersprachlicher Faktoren angeht, hinzuzufügen, da $\beta$ in inhaltbezogener Sicht vielfach das, was einfach Reaktion ist, als bedeutungsvoll überinterpretiert wird. So ist in Ich helfe DIR. vs. Ich unterstütze DICH. ein unterschiedlicher Inhaltswert qua Dativvs. Akkusativobjekt (also von der Verbsemantik abgesehen) zweifellos nicht nachweisbar (vgl. Helbig 1974, 159). Allerdings wird gelegentlich auf einen inhaltlichen Unterschied zwischen Dativ- und Akkusativobjekt bei Oppositionspaaren wie Er trat ihm auf den Fuß. / Er trat ihn auf den Fuß. hingewiesen (vgl. Wegener 1985, 166 ff). Er betrifft jedoch nicht das Abstempeln einer Person zum Gegenstand der Handlung vs. das Erfassen einer Person als gedanklich unangetastete Größe, sondern den Grad der Intentionalität der prädizierten Handlung: Die Akkusativkonstruktion wird eher als Ausdruck einer intentionalen Handlung interpretiert (Er hat mir unabsichtlich ins Gesicht geschlagen. wirkt akzeptabler als ?Er hat mich unabsichtlich ins 
Gesicht geschlagen.). Methodisch ist allerdings entscheidend, da 3 eine Formulierungsalternative zumindest vorstellbar ist. Unsinnig ist deshalb ein Befund wie der (vgl. ebd.), Akkusativkonstruktionen würden eher für den Ausdruck intensiverer Einwirkungen verwendet, Dativkonstruktionen für den weniger intensiver, wie in ${ }^{*}$ Er streicht sie übers Haar. / Er streicht ihr übers Haar. Das ist ein satzsemantisches Problem - der Rest ist Rektion.

\section{2. Über das Passiv}

Der Anstoß zu Beschäftigung mit dem Genus verbi ging nach Weisgerbers eigener Aussage von M. Wandruszka $(1961,46)$ aus, der in seiner kontrastiven Behandlung des Passivs schreibt: „Nicht nur 'der Mensch im Akkusativ' (Weisgerber) ist ein Kennzeichen der Sprache unserer Zeit, auch, 'der Mensch im Passiv', der Mensch, an dem sich ein Geschehen vollzieht." Die inhaltbezogene Betrachtung beginnt Weisgerber (vgl. $1963 \mathrm{~b}, 35 \mathrm{ff}$ ) mit einer kritischen Diskussion vorliegender inhaltlicher Kennzeichnungen der Diathesen, in der er sich gegen die nicht gerechtfertigte Definition von Passiv als 'Leideform' - und damit gegen die in dem Wandruszka-Zitat ausgedrückte Auffassung! - wendet. An Erbens (vgl. 1961, $30 \mathrm{ff}$ ) Analyse der Diathesen bezweifelt Weisgerber (1963 b, 43) deshalb mit plausiblen Argumenten, ,daß sich das Passivproblem inhaltlich von der geistigen Stellung des Objekts aus lösen läßt": Da Passivkonstruktionen auch bei objektlosen Verben möglich sind und in anderen Fällen von Passivierung das Objekt bestehen bleibt (Er bekommt die Zeitung gebracht.), ist es nach Weisgerber nicht gerechtfertigt, Intransivierung und Konversion der ursprünglichen Subjekte in Objekte zum zentralen Definitionspunkt des Passivs zu erheben. Weisgerbers eigener Versuch einer inhaltlichen Erfassung der Diathesen setzt deshalb bei der Rolle des Subjekts an. Zunächst wendet er sich gegen die Redeweise, das Passiv sei im Grunde eine Umkehrung des Aktivs (vgl. so noch Duden-Grammatik 1984, 117), denn ich werde gelobt sei nicht die Umkehrung von ich lobe, sondern allenfalls von du/er lobt mich/sie loben mich usw. Tatsächlich verallgemeinern die Vertreter der Auffassung vom Passiv als 'Konverse' in unzulässiger Weise Fälle wie $A$ sieht $B .-B$ wird von A gesehen., also Sätze mit transitivem Verb und explizitem Agens in beiden Versionen (vgl. Brinkmann 1962, 517 f). In „echter Opposition" zum Aktiv ist nach Weisgerber allen Passivvarianten nur eines gemeinsam: die „Ausschaltung des agierenden 'Täter'Subjekts“, „die sprachliche Fassung von Geschehnissen ohne herausgehobenen Urheber". Deshalb hält er die Kennzeichnung von Passiv als 'Leideform' für nicht angemessen: „Die nicht-aktivischen Sprachmöglichkeiten [...] setzen nicht an der Lage des 'Behandelten' an, sondern an der Stellung des 'Handelnden' " (Weisgerber 1963 b, 45 f). Die Opposition Aktiv - Passiv ist demnach für Weisgerber durch die Opposition ,verbale Forderung nach einem aufweisbaren 'Täter' " vs. „Belassen (oder Zurückversetzen) des Agens, des 'tatsächlichen' Trägers, unter den anders geordneten Komponenten eines Geschehens" zu charakterisieren (ebd., 46), die Agens-Aussparung ist die zentrale ,inhaltliche Eigenart" des Passivs. - In der leistungbezogenen Betrachtung (vgl. ebd., $48 \mathrm{ff}$ ) kommt einer sprachgeschichtlichen Argumentation besondere Bedeutung zu: Für Weisgerber impliziert die im idg. Verb „,so stark ausgebaute Täter-Diathese“ den Z Zwang, „das Geschehen immer auf einen identifizierbaren Urheber zurückzuführen“ und ,den bekannten oder vermuteten Urheber immer zu nennen“. Diese Form sprachlicher Weltgestaltung werde dort zu einer Überforderung, wo das Anheften des Verbalgeschehens an eine bestimmte Größe keinen rechten Sinn mehr mache (vgl. der Wind weht). Die Sprachgemeinschaft spüre solche Zwangslagen, was zu neuen Verfahrensweisen führe - wie für das Deutsche in ahd. Zeit geschehen (vgl. ebd., $50 \mathrm{f}$ ). Die „Kernleistung“ des Passivs definiert Weisgerber (ebd., 52) deshalb abschließend so: ,In einem durch das idg. persönliche Aktiv beherrschten verbalen Denkkreis werden Verfahrensweisen geschaffen, die ein Begreifen von Geschehnissen gestatten, das nicht täterbezogen abläuft." - Grundsätzlich stimmen die Grammatiken in dieser Charakterisierung der besonderen Leistung des Passivs als Möglichkeit, ein Geschehen ohne herausgehobenen Verursacher zu fassen, überein (vgl. u. a. Heidolph et al. 1981, 540 f, die die Diathesen mittels der Kategorien 'täterbezogen' und 'nicht täterbezogen' beschreiben). Und tatsächlich macht die Sprachgemeinschaft von der Agens-Aussparung beim Passiv auch regen Gebrauch (vgl. Schoenthal 1976, 124). Dennoch erfassen Kategorien wie 'nicht täterbezogen' bestenfalls die halbe Wahrheit, denn der 'Täter', das Agens, kann ja in der Passivergänzung oder an anderer Stelle im Satz, z. B. als Genitivattribut (vgl. Der Angriff der Hamburger wird nun aus der eigenen Dek- 
kung aufgebaut.; Schoenthal 1976, 130) genannt werden. Unter diesem Aspekt stellt sich die Frage nach Inhalt und 'Leistung' des Passivs erneut, wobei sich ein Ausgehen vom dreigliedrigen werden-Passivsatz empfiehlt (vgl. Schoenthal 1987, 161 ff): Für diesen gilt lediglich, daß das Agens nicht als grammatisches Subjekt auftritt. 'Nicht täterbezogen' muß deshalb, enger als bei Weisgerber, im Sinne von 'das Agens steht nicht in der Rolle des grammatischen Subjekts' definiert werden, womit über das Vorkommen des Agens in anderen Positionen des Satzes noch nichts gesagt ist. Betrachtet man die Verhältnisse auf Text-Ebene, so stellt man fest, daß die das Agens ausdrückende Passivergänzung häufig gerade Rhema des Passivsatzes ist, also einen „höheren Mitteilungswert“ besitzt als das Subjekt (ebd., 168), wie im Beispiel (1) Der Präsident schlägt den Kanzler vor. Der Kanzler wird vom Parlament gewählt. (vgl. Eisenberg 1986, 142 f). Für die Verwendung von werdenPassivsätzen gilt somit: Sie ermöglichen bzw. bewirken (a) Agenslosigkeit, (b) Thematisierung des Objekts/Patiens, (c) Rhematisierung des Prädikats, (d) Rhematisierung der Passivergänzung/des Agens, (e) Rhematisierung von Prädikat und Passivergänzung/Agens. Da, wie die Satzfolge (2) Der Präsident schlägt den Kanzler vor. Den Kanzler wählt das Parlament. zeigt, auch Aktivsätze zur Rhematisierung des Agens geeignet sind (wenngleich (2) weniger 'natürlich' klingt als (1), weil die Satzgliedfolge markiert ist, vgl. Eisenberg, ebd.), reduziert sich der Kontrast Aktiv-Passiv in kommunikativ-pragmatischer Hinsicht auf die Verwendung (e): „Das Passiv ist nicht besser als das Aktiv zur Rhematisierung des Agens geeignet, sondern der dreigliedrige werden-Passivsatz ermöglicht [...] vor allem die Rhematisierung von Prädikat und Agens, wie sie im Aktiv unmöglich ist" (Schoenthal 1987, 175; Hervorhebg. von mir; J. D.). - Die wirkungbezogene Betrachtung der Diathesen ist bei Weisgerber (vgl. 1963 b, 53 ff) Programm geblieben. Welche „Folgen für die Gestaltung des menschlichen Lebens" er ihnen zusprechen würde, wird in Andeutungen dort sichtbar, wo Weisgerber sich auf H. Hartmann bezieht, der die „Ausprägung eines spezifischen starken 'Passivs' " im Irischen mit einer Denkform des "Glaubens an eine Allkraft" in Verbindung bringt. Derartige Spekulationen mögen attraktiv sein, empirisch nachprüfbar sind sie jedenfalls nicht.

\section{3. Über Satzbaupläne}

Eine syntaktische Analyse, die für alle Stufen der inhaltbezogenen Grammatik durchgeführt ist, liegt mit Weisgerbers (vgl. 1962 b) „ganzheitlicher Behandlung eines Satzbauplanes" anhand von Er klopfte seinem Freunde auf die Schulter. vor. Der Begriff 'Satzbauplan' soll, im Unterschied zu Begriffen wie 'Satztyp' oder 'Satzmodell', die energetische Perspektive ausdrücken: nach einem SatzBauplan wird in einer Sprache gearbeitet, und Weisgerber insistiert (in schöner Nähe zum ungeliebten Chomsky) auf den „Möglichkeiten zu Satzformulierungen" als Gegenstand der Sprachbetrachtung - es geht nicht um die bereits formulierten Sätze. Der eigentlich energetische Zug kommt allerdings erst durch die syntaxtheoretische Grundthese zum Ausdruck: Jeder auftretende individuelle Satz ist nicht einfache 'Wiedergabe' von 'objektiven Geschehnissen', sondern Prägung von Geschehen zu sprachlicher Bewußtheit" (ebd., 7). Die methodische Schwierigkeit dieses Ansatzes liegt zum einen in den „scheinbar [?]“ unbegrenzten Realisierungen von Sätzen. Zum anderen sieht Weisgerber selbst aber auch das Problem, an dem letztlich das Programm der inhaltbezogenen Syntax scheitert: was nämlich bleibt an Inhaltlichem, wenn man von der „einmaligen Wortfüllung“ des Satzes abstrahieren muß - also von der Satzbedeutung, sowie von der „einmaligen Situation" der Satzrealisierung - und damit auch von der Äußerungsbedeutung? (ebd., 5) Es spricht für Weisgerbers wissenschaftlichen Scharfblick, die Probleme gesehen zu haben. Aber er hat sich das Aporetische seiner inhaltbezogenen Syntax nicht eingestehen wollen. - Die lautbezogene Betrachtung arbeitet vor allem die ,rhythmisch-melodische Gliederung" des Satzbauplanes und die Abfolge der Satzglieder heraus. Die inhaltbezogene Betrachtung versucht, Gruppen von inhaltlich zusammengehörenden Phänomenen aufzustellen. Für die Syntax liegt nach Weisgerber ein Ansetzen bei solchen „Aufgabenbereichen" wie Befehl, Frage, Wunsch oder Aussage nahe - modern gesprochen also: bei den Sprechakttypen -, und diese sind nach seiner Auffassung im Satz durch die finiten Verben realisiert. Im vorliegenden Fall haben wir es nach Weisgerber so gesehen mit einem „Aussagesatz" zu tun - einer, wie er meint, allerdings noch zu groben Kategorie, die er mit Brinkmann in „Vorgangssatz, Handlungssatz, Urteilssatz" usw. ausdifferenzieren möchte 
(vgl. unten, Abschnitt 5.). Genauer ist nach Weisgerber Er klopfte seinem Freunde auf die Schulter. als "zugewandter Bestätigungssatz“ zu klassifizieren, dessen "geistige Struktur" sich als Grundsituation beschreiben läßt, ,in der ein Geschehen durchaus erscheint, als Tätigkeit eines Agenten, jedoch so, daß es weder als zielgerichtete Handlung noch als in sich gekehrte Beschäftigung noch als isolierte Aktivität erscheint"; wobei die Aktivität sich vielmehr ,gemäß ihrem Schauplatz bezogen [zeigt] auf einen Teilnehmer, dessen Interessen durch diese Betätigung mitbetroffen sind und auf dessen absichtliches oder tatsächliches Einbeziehen sie hinausläuft" (ebd., 17). 'Zugewandt' steht dabei, nach Weisgerber, für die Beobachtung, daß bei diesem Satzbauplan die Betätigung ,einem Wesen zugewandt" ist (vgl. (a) *Die Sonne schien den Bergen auf die Spitze.). Die Rede von der nicht zielgerichteten Handlung leitet sich von Beobachtungen her wie dem Unterschied zwischen Er trat mich auf den $F u \beta .=$ zielgerichtet vs. Er trat mir auf den Fu $\beta .=$ nicht zielgerichtet (vgl. dazu oben, Abschnitt 4.1.). - Bei genauerem Hinsehen zeigt sich, daß Weisgerbers inhaltbezogene Analyse weder völlig zutreffend noch vollständig ist. Die Begriffe 'zugewandt' und 'Tätigkeit' decken einen Fall wie (b) Der Regen tropft mir auf den Hut. nicht ab. Daß sich das in einem Satzbauplan mit Pertinenzdativ auftretende präpositionale Objekt nur auf Körperteile $(F u \beta)$ und Accessoires (vgl. Hut in (b)) beziehen kann (so die DudenGrammatik 1973, 486 f), ist im übrigen auch nicht richtig, wie (c) Die Katze läuft mir über den Weg. zeigt. Die detaillierte Analyse ergibt hingegen folgende satzsemantische Bedingungen (vgl. v. Polenz 1969, $151 \mathrm{ff}$ ): Das Dativobjekt bezeichnet ein Lebewesen (auch metaphorisch: (d) Die Lokomotive fuhr dem Schnellzug in die Flanke.). Das präpositionale Objekt muß in Relation zum Dativobjekt gesehen werden und ist nicht auf Körperteile (vgl. (b)), nicht einmal auf eine Teil-von-Relation eingeschränkt (vgl. (c)), sondern steht für ein allgemein gefaßtes 'Teilhaben' (in dem Sinne, wie man auch in (c) von meinem Weg sprechen kann). Schließlich ist für das Dativobjekt die semantische Bedingung „Partizipieren des Lebewesens am Vorgang", den das Prädikat ausdrückt, anzusetzen, um einen Satz wie (a) auszuschließen (vgl. ebd., 166; vgl. auch Wegener 1985, $88 \mathrm{ff}$ für eine Ausgrenzung der Pertinenzrelationen). - Die leistungbezogene Betrachtung fragt nach dem sprachlichen Zugriff durch diesen Satzbau- plan, jedoch zeigt sich, daß diese Analyse gegenüber der inhaltbezogenen nicht recht weiterführt - eine methodische Schwäche, die mehrfach bei Weisgerber sichtbar wird (vgl. Dittmann 1980, 55). - Für die wirkungbezogene Betrachtung greift Weisgerber (vgl. 1962 b, 30 ff) letztlich auf Überlegungen aus der inhaltbezogenen und leistungbezogenen Betrachtung zurück, denen zufolge ein Plan wie in Er trat ihm auf den Fuß. die betroffene Person als ,beteiligte, aber gedanklich unangetastete Größe“, ein Plan wie in Er trat ihn auf den Fuß. die betroffene Person dagegen als "Gegenstand einer Handlung" abstempelt. Wir haben oben, vgl. Abschnitt 4.1., bereits gesehen, daß diese Opposition so nicht haltbar ist. Nach Weisgerber aber gilt: „Die deutsche Sprache hält Satzbaupläne bereit, die geistige Situationen schaffen, in denen ein in eine Tätigkeit einbezogenes Wesen entweder als eigenständig beteiligtes oder als einer Absicht unterworfenes geistig gefaßt wird." (ebd., 33). Die Sprecher müssen folglich zwischen diesen beiden Sichtweisen wählen, sie müssen ,entscheiden, in welcher Weise sie mit diesem Wesen gedanklich (und in der Folge auch praktisch) verfahren". Weisgerber stellt also hier ganz im Sinne seiner sprachtheoretischen Vorgaben die starke Hypothese auf, daß ein Satzbauplan qua impliziertem Denkmuster Folgen für die gesellschaftliche Praxis haben kann. Die ganzheitliche Betrachtung des Satzbauplanes zeigt nicht etwa nur die Grenzen (die werden auch auf dem Gebiet der Morphologie und Wortbildung schon deutlich), sondern die prinzipielle Problematik der inhaltbezogenen Grammatik überhaupt: Aussagen über den Inhalt sprachlicher Ausdrucksmittel, verstanden als je einzelsprachlich festgelegte Bedeutung auf der Ebene der Langue, setzen die Abstraktion von Verwendungskontexten voraus, denn sie sollen ja für alle Verwendungskontexte gelten. Nun ist es wohl möglich und sinnvoll, die Frage nach der 'wörtlichen Bedeutung' von Sätzen zu stellen. Jedoch nimmt diese Beschreibung zwangsläufig Bezug auf die konkrete inhaltliche Füllung der Sätze (zumindest in Form des Bezuges auf die Proposition). Die inhaltliche Beschreibung von Satztypen dagegen ist prinzipiell nicht möglich, denn die Abstraktion vom konkreten Satz kann immer nur hinsichtlich einzelner Aspekte erfolgen, nicht jedoch so, daß sie zu einem 'Inhaltschema' der von Weisgerber angestrebten Art führt: Erhalten bleibt de facto die Verallgemeinerung nicht nur auf 
einen Kreis von bestimmten Verben hin, die in diesen Sätzen im Prädikat auftreten, sondern auch auf bestimmte Typen von Subjekten und Objekten hin: was vielleicht bei einer bestimmten Interpretation der Äußerung einer Instanz des Satzes Er klopfte seinem Freunde auf die Schulter. zutrifft (der Freund als vollwertige Person betrachtet usw.), wird in bezug auf andere Aktanten schlicht falsch. Die vorgebliche Beschreibung eines Satztyps entpuppt sich als Resultat der Verallgemeinerung von Befunden, die für Vorkommen einzelner Sätze dieses Typs in bestimmten Kontexten gelten mögen - die Beschreibung ist einerseits verallgemeinernd, andererseits zugleich tautologisch (vgl. Heeschen 1972, 67). Deshalb müssen auch Gippers (vgl. 1970, $37 \mathrm{f})$ erneute Bemühungen um diesen Satzbauplan scheitern, auf die hier nicht näher eingegangen werden kann.

\section{Satzmodelle als (auch) inhaltlich definierte Satztypen}

Weisgerber (vgl. u. a. 1962 b, 19f) verweist in seinen Arbeiten zum Thema 'Satzbaupläne' mehrfach auf $\mathrm{H}$. Brinkmanns Theorie der „Satzmodelle“ und konstatiert, Brinkmanns "Grundmodelle“ warteten darauf, ,in ausdrücklich energetischen Überlegungen fortgeführt zu werden“. Nach seiner Auffassung gehören sie auf eine höhere Abstraktionsstufe als die Satzbaupläne; daß dies nur bedingt zutrifft, macht ein Blick auf Brinkmanns Theorie deutlich. - Brinkmann (1962, $455 \mathrm{ff})$ faßt den Satz als ,geistige Einheit“ auf, wobei er sich neben der Intonation auch auf die Möglichkeit der Pronominalisierung bezieht (Willst du im Sommer mit uns verreisen? $D A S$ weiß ich noch nicht.). Dem Satz eignet eine „Satzintention“, die durch das finite Verb definiert ist (Aussage über die Person, die Diathesen, die "Zeitauffassung" $=$ Tempus, Einstellung des Subjekts zum „dargestellten Proze $\beta^{\prime \prime}=$ Modus). Ein erster Ansatz zur Typisierung von Sätzen ergibt sich aufgrund der unterschiedlichen Stellungsmöglichkeiten des finiten Verbs: Sätze mit „Zweitstellung“, der Normalstellung, „dienen dem Austausch von Mitteilungen. Sie erlauben situationsfreies Reden, d.h. einen Gedankenaustausch, der unabhängig von der jeweiligen Situation ist“. Sie heißen deshalb „Mitteilungssätze“ (ebd., 473). Sätze mit „Spitzenstellung“ dagegen sind ,an eine Situation gebunden und auf den Partner gerichtet", sie heißen deshalb
„Partnersätze“. Prototypisch gehören die Aufforderung und die Satzfrage hierher (vgl. ebd., $473 \mathrm{ff}$ ). D. h. Brinkmann unterscheidet in diesem Kontext nicht durchgängig zwischen formal definierten Satztypen und Sprechakttypen: Aufforderungen als Sprechakte können selbstverständlich mit ganz unterschiedlichen sprachlichen Mitteln realisiert werden (vgl. Dittmann 1981, $137 \mathrm{ff}$ ). Es ist heute klar, daß Brinkmanns Auffassung vom Verhältnis zwischen Grammatik und 'Pragmatik' zu eng ist, wenn er schreibt: „Die Stellung der Personalform, bei der die Satzintention liegt, unterscheidet Arten des Satzes nach ihrer Aufgabe für die Kommunikation" (Brinkmann 1962, 508). Bemerkenswert bleibt aber, daß er jedenfalls die Fragestellung, Jahre vor der sog. pragmatischen Wende in der Linguistik, stets im Blick hat. Sätze mit „Endstellung“" (bzw. „Spätstellung“) des finiten Verbs schließlich haben den Status von Satzgliedern, weshalb sie auch keine eigene Satzintention aufweisen (vgl. ebd., $478 \mathrm{ff}$ ). Die Analyse der "Satzmodelle“ beruht zunächst wieder auf einer grammatisch-formalen Einteilung, nämlich nach der Art des Prädikates (verbales, adjektivisches, substantivisches). Es ergeben sich vier „Grundmodelle“: Verbalsatz, Adjektivsatz, Substantivsatz, wobei derselbe Sachverhalt auf verschiedene Weisen dargestellt werden kann (Sein Kommen hat mich überrascht. / Sein Kommen war für mich überraschend. / Sein Kommen war für mich eine Überraschung.). Die Ausdifferenzierung des Grundmodells 'Verbalsatz' geht von der Unterscheidung zwischen transitivem und intransitivem Verb als Prädikat aus, wobei Brinkmann (ebd., 517) 'transitiv' eng definiert, i.S. einer zweiseitig-umkehrbaren Beziehung, die inhaltlich einen ,übergreifenden

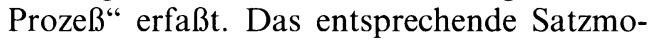
dell heißt „Handlungssatz“. Ist das Prädikat ein intransitives Verb (so daß keine ,zweiseitige Beziehung“ aufgebaut wird), werden ,immanente Prozesse" bezeichnet - Brinkmann spricht von „Vorgangssatz". Interessant ist, $\mathrm{da} ß$ diese Unterscheidung in der Tat zunächst einem recht abstrakten Kriterium folgt, nämlich einer Differenzierung zwischen transitivem und intransitivem Verb, relational definiert. Insoweit ist Weisgerbers Einschätzung des unterschiedlichen Status' seiner Satzbaupläne und der Brinkmannschen Satzmodelle korrekt. Allerdings wagt sich Brinkmann bei der Diskussion der Begriffe 'Vorgangs-' und 'Handlungssatz' denn doch an eine inhaltliche Definition, die nah an eine inhaltbezogene 
Betrachtung im Weisgerberschen Format heranführt. Zunächst sagt er, diese Bezeichnungen seien ein Behelf, weil sie nur je einen exemplarischen Fall des jeweiligen Satzmodells darstellten (vgl. ebd., 520). Dann aber führt er näher aus, bei einem Vorgang werde das Subjekt als Ort, bei einer Handlung als verantwortlicher Urheber des verbalen Prozesses aufgefaßt: ,Als Vorgangssatz fassen wir alle Fälle, in denen sich im verbalen Prozeß das Dasein oder das Sosein des Subjekts bekundet; als Handlungssatz alle Fälle, in denen das Subjekt über Dasein oder Sosein des Objekts bestimmt" (ebd., 520). In dem Satz Man brachte Licht und das Gepäck kam. (Th. Mann) ist beides realisiert: die Handlungsperspektive in bezug auf das Licht und die Vorgangsperspektive in bezug auf das Gepäck. Daß tatsächlich beides gebracht werden muß - Koffer bewegen sich nicht von selbst -, zeigt, „daß es nicht auf die tatsächliche Sachlage ankommt“, denn auch, was „sein Sosein erst durch den Menschen empfängt, kann vorgestellt werden, als ob es von sich aus sein Sosein zu verändern vermöge", was „eigentlich Handlung ist, wird als Vorgang dargestellt". Der Primat der Sehweise gegenüber der Sachlage kennzeichnet Brinkmanns inhaltliche Analysen also genau so wie die Weisgerbers. Nur führt, so Helbig (1974, $160 \mathrm{f}$ ), bei Brinkmann die Mischung aus struktureller (transitives vs. intransitives Verb) und semantischer Kategorisierung zu einem gelegentlich kontraintuitiven Gebrauch der Begriffe „Handlungs-“ und „Vorgangssatz" (vgl. Helbig 1974, 160 f): Handlungssätze drücken nicht immer eine Handlung im realen Sinne aus (z. B.: Er erleidet eine Krankheit.), ebensowenig wie Vorgangssätze immer Vorgänge im realen Sinne ausdrücken (z. B. Er liegt im Bett.). Durch diese Spannung zwischen struktureller und semantischer Ebene werden Brinkmanns Kategorien schwer handhabbar, zumal es ihm selbst offenbar mehr auf die inhaltliche als auf die formale Betrachtungsweise in der Syntax ankommt (vgl. die zusammenfassende Beschreibung von Handlungs- und Vorgangssatz bei Brinkmann 1962, 522 , und die jeweiligen Subkategorisierungen ebd., $522 \mathrm{f}$ ). - Diese Spannung kommt in der Behandlung von Adjektiv- und Substantivsatz (vgl. ebd., $559 \mathrm{ff}$ ) besonders deutlich zum Ausdruck: Nach Brinkmann kann „derselbe Sachverhalt" einstellig, mittels Adjektiv, und zweistellig, mittels Substantiv dargestellt werden: (a) Diese Tat ist verbrecherisch. / (b) Diese Tat ist ein Verbrechen. In (a) werde die
Ablehnung einer Tat zum Ausdruck gebracht, in (b) die Tat klassifiziert. Selbstverständlich ist diese Unterscheidung von „qualifizierendem" Adjektivsatz und „klassifizierendem“ Substantivsatz überhaupt kein morphosyntaktisches, sondern ein rein lexikalisch-semantisches Problem (vgl. den 'qualifizierenden' Substantivsatz Dieser Brief ist eine Unverschämtheit.). Auch hier, wie schon bei Weisgerber, steht die semantische Analyse morphosyntaktischer Strukturen vor der Schwierigkeit, von der konkreten Satzbedeutung in Richtung auf 'Konstruktionsbedeutungen' abstrahieren zu müssen: ein Unterfangen, das scheitert, weil ein Rest an konkreter Satzbedeutung zwangsläufig in jeder noch so weit getriebenen Abstraktion erhalten bleibt, soll diese nicht leer sein. Dieser Rest aber verhindert, was eigentlich anzustreben wäre: Er reduziert die Anwendbarkeit der Analyse auf semantisch bestimmte Teilklassen der morphosyntaktischen formalen Klassen, um die es eigentlich geht.

\section{Der Text als Gegenstand inhaltbezogener Sprachforschung}

Ob H. Glinz der Richtung 'inhaltbezogene Grammatik' zuzuordnen ist, ist umstritten (vgl. Szemerényi 1982, 275 f; Dittmann 1980, $157 \mathrm{f})$. In seiner „Inneren Form des Deutschen" sieht Glinz (1952, 13 f) jedenfalls ausdrücklich keinen Versuch, aufzuzeigen, wie die Sprache „zur Erforschung und Darstellung der menschlichen Lage und des menschlichen Daseins dienen kann und welche Ergebnisse damit gewonnen werden" - das sind für ihn philosophische Fragen. Sein Anliegen ist vielmehr, ,die Struktur der deutschen Sprache in höherem Grade durchsichtig zu machen, als es in der bisherigen Grammatik geschah". Zu diesem Zwecke entwickelt er „Proben“, die, auf Texte (!) angewendet, Satzglieder als je notwendige Bestandteile deutscher Sätze sowie syntaktisch-funktionell identische Einheiten liefern. So findet er durch Ersatz- und Verschiebeprobe ein Satzglied heraus, das immer 'einwortig' ist, nur durch seinesgleichen ersetzt werden kann und einen festen Pol im Satzbau bildet, indem es stets an zweiter, letzter oder erster Stelle auftritt. Deshalb nennt er es „Leitglied“ (gemeint ist also das finite Verb). Die drei ,Stellungstypen“ nennt er zunächst „Kernform, Spannform und Stirnform", um nicht schon Inhaltliches bzw. Funktionales zu präjudizieren. 
Doch auch die Bestimmung der ,Inhaltswerte" dieser Satztypen reicht nicht im entferntesten an die hermeneutischen Eskapaden Weisgerbers heran. Glinz (1952, $422 \mathrm{ff})$ begnügt sich mit dem Befund, der Wert des Spannsatzes sei mit ,anzuschließen an einen anderen Ausdruck" anzugeben (vgl. Warum kamest du nicht? Weil es mich gereut hat!). Die häufigste Verwendung sei deshalb die als Nebensatz. Der Stirnsatz habe den Wert „,besondere Ladung, besondere Spannung“, wie sie im Ausruf (Hat er endlich Schluß gemacht!) und der Frage zum Ausdruck komme. Demgegenüber sei der Kernsatztyp (Er hat endlich Schluß gemacht.) als Normalform, sein Wert als „gewöhnlich“ zu bestimmen. Wichtig ist im übrigen, daß Glinz wie kaum ein Grammatiker vor ihm, von der gesprochenen Sprache ausgeht, die Stimmführung also stets als einen Faktor der Satzanalyse mit einbezieht (vgl. ebd., 425). Glinz sieht das Erfassen von sprachlichen Inhalten als Prozeß der Interpretation, die die (conscience individuelle) zum Ausgangspunkt hat. Das entscheidende methodische Problem ist nun, daß es ja formal gewonnene Einheiten sind, die durch Interpretation mit Inhalt gefüllt werden sollen (vgl. ebd., $57 \mathrm{f}$ ); damit präjudiziert die ausdrucksseitig ansetzende Probe jeweils eine Gliederung in sprachliche Einheiten, die in der Interpretation als zugleich inhaltliche Einheiten aufgefaßt werden (vgl. die Kritik bei Helbig 1974, 223; vgl. schon Weisgerber 1953/ 54, 117). Dieses Problem hat Glinz gesehen und thematisiert, und dieses Problem hat ganz offensichtlich sein Interesse an der ,inhaltbezogenen Sprachforschung“ (er spricht nicht von 'inhaltbezogener Grammatik') geweckt: „Inhaltbezogene Sprachforschung setzt sich zum Ziel, die Sprachinhalte [...] zu erforschen, auch dort, wo diese rein geistige Sprachstruktur nicht parallel ist zur formalen, an den Lautungen erkennbaren Struktur und man daher nicht mehr, wie man es sonst als Wissenschaftler immer tun muß [!], von dieser formalen Struktur ausgehen und dadurch die nötige Objektivität erreichen kann." (Glinz 1965, 7; vgl. $1962 \mathrm{a}, 18)$. Als neuen Weg schlägt er deshalb eine ,'Verstehens-Analyse' ausgewählter Texte" vor, die erarbeitet, was „Sprache und sprachliches Handeln“ für die Menschen leisten (1962 b, 11). Ein Ziel dieser Analysen ist der Versuch, eine Typologie elementarer sprachlicher Handlungen zu entwikkeln (vgl. ebd., Kap. 1.), Ansätze, die Glinz (vgl. 1973; 1978) auch in expliziter Auseinandersetzung mit Theorien sprachlichen Han- delns fortführt. Der Unterschied zu Weisgerbers ganzheitlicher Analyse eines Satzbauplanes läßt sich an folgendem Beispiel demonstrieren (vgl. Glinz 1965, 42 ff): Die ,strukturalistische Analyse“ des Satzes Es tut nichts. ergibt als "Gliederungsplan" die Folge „Grundgröße, Personalform, Zielgröße“. Die Inhaltsanalyse zielt auf das „Gemeinte“ des Satzes, d. h. auf die Satzbedeutung dieses Satzes, nicht etwa des Gliederungsplanes als Typ. Das Gemeinte erschließt sich durch Vergleich mit quasi gleichbedeutenden Sätzen (wie ES schadet nichts.) als „tröstendes Zureden“, jedoch ist es aus der „Morpho-Kette nicht bindend herauszulesen": Wenn es als auf ein „personales Wesen“ referierend (z. B. das Kind) und tut im Sinne von arbeiten verstanden wird, ergibt sich ein entsprechend anderes Gemeintes. - Auch Glinz' spätere Arbeiten zur deutschen Grammatik bleiben in der Tradition der „Inneren Form“ an Tests orientiert und korpusbezogen (vgl. etwa die Satzgliedbestimmungen in Glinz 1971, 33 ff), wobei er zunehmend die Terminologie der traditionellen (und zugleich der modernen) Grammatik übernimmt ('Objekt' statt 'Zielgröße' usw.).

\section{Abschließende Würdigung}

Die zusammenfassende Würdigung der inhaltbezogenen Grammatik unter dem Aspekt der morphosyntaktischen Fragestellungen soll nicht die in den einzelnen Abschnitten geäußerte Kritik wiederholen. Es sei lediglich noch einmal in aller Kürze auf die grundlegende Problematik und den prinzipiellen methodischen Irrtum eingegangen, die die inhaltbezogene Grammatik scheitern ließen. Die grundlegende Problematik besteht in dem bereits von Weisgerber selbst erkannten komplexen Verhältnis von Struktur morphosyntaktischer Ausdrucksmittel einerseits und deren Bedeutung bzw. der Satzbedeutung andererseits. Glinz (vgl. 1978, $112 \mathrm{ff}$ ) hat das mit aller wünschenswerten Klarheit anhand des Satzes (a) Jetzt reicht es dann. dargestellt. $\mathrm{Zu}$ diesem Satz gibt es eine Fülle ,phonomorphischer Varianten“" (wie Jetzt reicht's dann., dialektale Varianten usw.), bei identischer „grammatischer Struktur“. Umgekehrt kann aber die Bedeutung, mit der (a) in einem bestimmten Kontext geäußert werden kann, auch mit ganz anderen ,morphostrukturellen" Mitteln ausgedrückt werden: (b) Jetzt ist es dann soweit., (c) Ich bin gleich soweit. usw. (vgl. Glinz 1978, 114 f). Daraus zieht Glinz den Schluß: „Die Morphostruktur [...] kann 
einen bloßen grammatischen Mechanismus darstellen, der für die Korrektheit der betreffenden $\ddot{A}$ ußerung einzuhalten ist, der aber für die Gesamtbedeutung [...] und damit für das Verstehen und den sachlichen Kommunikationserfolg keine Rolle spielt." Mancher Linguistin und manchem Linguisten wird diese Formulierung zu radikal sein, denn die Suche nach Relationen zwischen der morphosyntaktischen Form von Sätzen, Satzbedeutungen und, z. B. Sprechakttypen, die mit der 苻ßerung von Instanzen von Sätzen vollzogen werden können, scheint so völlig aussichtslos nicht zu sein (vgl. u. a. Motsch/Viehweger 1981, $128 \mathrm{ff}$ ). Jedenfalls lehrt die Einsicht in die Komplexität dieser Relationen (vgl. u. a. Bierwisch 1979, 65 ff) aber eindeutig, daß zwischen Morphosyntax, Semantik und, z. B., der Beschreibung von Sprechakttypen kategorial zu unterscheiden ist. Deshalb ist die zweite Basisannahme der inhaltbezogenen Grammatik, mit der gerade die kategoriale Ununterscheidbarkeit von Morphosyntax und Semantik unterstellt wird, nicht haltbar, und das ist ausschlaggebend für das Scheitern der inhaltbezogenen Grammatik. Damit ist bereits impliziert, da $\beta$ auch die zentrale methodologische Vorgabe prinzipiell irrig ist: die ganzheitliche Sprachbetrachtung führt die Analyse von Inhalt, Leistung und Wirkung morphosyntaktischer Ausdrucksmittel immer wieder auf die aus einer gestaltbezogenen Betrachtung gewonnenen Größen zurück, dergestalt daß, etwa bei Brinkmanns 'Satzmodellen', die quasi formal gewonnenen Strukturen letztlich doch mit einem, wie immer abstrakten, Inhalt in Beziehung gebracht werden (vgl. oben, Abschnitt 5.). Das ist methodisch genauso verwerflich wie Weisgerbers Kurzschluß von den 'Aufgabenbereichen' (sprich: Sprechakttypen) über die Stellung des finiten Verbs auf inhaltlich bestimmte Satzbaupläne (vgl. oben, Abschnitt 4.3.). - Was von der inhaltbezogenen Grammatik bleibt, ist nach meiner Einschätzung zweierlei: Erstens haben die Vertreter der inhaltbezogenen Grammatik mit dem Versuch einer 'ganzheitlichen' Sprachbetrachtung ein Werk begonnen, das auf gesicherterer sprach- und grammatiktheoretischer Basis und mit überdachter Methode fortgeführt werden sollte. Ansätze, die morphosyntaktische, semantische und sprechakttheoretische (oder andere 'pragmatische') Kategorien auf je identische Gegenstandsbereiche anwenden, gehen da vermutlich in die richtige Richtung (vgl., neben Bierwisch 1979, u.a. Růžǐčka 1983). Zweitens bleibt die in der ersten Basisannahme der inhaltbezogenen Grammatik formulierte sprachtheoretische Position, die auf eine zunächst härter, später weniger hart (vgl. Weisgerber 1973, 19 f) - formulierte Determinismushypothese hinausläuft, eine Herausforderung für alle, die anderer Auffassung sind, ebenso wie Weisgerbers Bestimmung des Verhältnisses von Sprache, Sprachgemeinschaft und Individuum (vgl. Dittmann 1980, $50 \mathrm{f} ; 59)$ - ein Thema von bleibender Aktualität.

\section{Literatur}

Bierwisch, Manfred. 1979. Wörtliche Bedeutung eine pragmatische Gretchenfrage. Sprache und Pragmatik, Lunder Symposium 1978, hrsg. von Inger Rosengren, 63-85. (Lunder germanist. Forschungen 48). Lund.

Brinkmann, Hennig. 1962. Die deutsche Sprache. Gestalt und Leistung. (Sprache und Gesellschaft, Grundlegung 1). Düsseldorf.

Chomsky, Noam. 1977. Essays on form and interpretation. (Studies in Linguistic Analysis). Amsterdam.

-. 1980. Rules and representations. Oxford.

-. 1981. Lectures on government and binding. The Pisa Lectures. (Studies in Generative Grammar 9). 4th edn. 1986. Dordrecht, Riverton.

Dittmann, Jürgen. 1980. Sprachtheorie der inhaltbezogenen Sprachwissenschaft. Teil 1/Teil 2. Deutsche Sprache 8. 40-74, 157-76.

-. 1981. Konstitutionsprobleme und Prinzipien einer kommunikationsorientierten Grammatik. Dialogforschung. Jahrbuch 1981 des Instituts für deutsche Sprache, hrsg. v. Peter Schröder \& Hugo Steger, 135-77. (Sprache der Gegenwart 54). Düsseldorf.

Duden-Grammatik. 1973. Duden-Grammatik der deutschen Gegenwartssprache. (Der Große Duden, 4) 3. Aufl. Mannheim.

-. 1984. Duden-Grammatik der deutschen Gegenwartssprache. (Der Große Duden, 4). 4. Aufl. Mannheim.

Eisenberg, Peter. 1986. Grundriß der deutschen Grammatik. Stuttgart.

Erben, Johannes. 1961. Abriß der deutschen Grammatik. 4., neubearb. Aufl. Berlin, DDR.

Geckeler, Horst. 1971. Strukturelle Semantik und Wortfeldtheorie. München.

Gipper, Helmut. 1970. Der Satz als Steuerungs- und Regelsystem und die Bedingungen der Möglichkeit seines Verstehens. Studien zur Syntax des heutigen Deutsch, Festschrift für Paul Grebe, 26-44. (Sprache der Gegenwart 6). Düsseldorf. 
-. 1974. Inhaltbezogene Grammatik. Grundzüge der Literatur- und Sprachwissenschaft. Bd. 2: Sprachwissenschaft, hrsg. v. H. L. Arnold \& V. Sinemus, 133-50. München.

-, und Peter Schmitter. 1975. Sprachwissenschaft und Sprachphilosophie im Zeitalter der Romantik. Current Trends in Linguistics. Vol. 13, Historiography of Linguistics, hrsg. v. Thomas A. Sebeok, 481 - 606. The Hague, Paris.

-, und Hans Schwarz (Hg.). 1962 ff. Bibliographisches Handbuch zur Sprachinhaltsforschung. Teil I, Schrifttum zur Sprachinhaltsforschung in alphabetischer Folge nach Verfassern mit Besprechungen und Inhaltshinweisen. Bde. 1-4. (Wissenschaftliche Abhandlungen der Arbeitsgemeinschaft für Forschung des Landes Nordrhein-Westfalen, $16 \mathrm{a}$; ab Bd. 2: Abhandlungen der rheinisch-westfälischen Akademie der Wissenschaften, 16a). Opladen.

Glinz, Hans. 1952. Die innere Form des Deutschen. Eine neue deutsche Grammatik. Bern, München.

-. 1962 a. Sprache und Welt. (Duden-Beiträge 6). Mannheim.

-. 1962 b. Ansätze zu einer Sprachtheorie. (Beihefte zu Wirkendes Wort 2). Düsseldorf.

-. 1965. Grundbegriffe und Methoden der inhaltbezogenen Text- und Sprachanalyse. (Sprache und Gemeinschaft, Grundlegung 3). Düsseldorf.

-. 1971. Deutsche Grammatik II. (Studienbücher zur Linguistik und Literaturwissenschaft 3). Frankfurt/M.

- . 1973. Textanalyse und Verstehenstheorie I. (Studienbücher zur Linguistik und Literaturwissenschaft 5). Frankfurt/M.

-. 1978. Textanalyse und Verstehenstheorie II. (Studienbücher zur Linguistik und Literaturwissenschaft 6). Frankfurt/M.

Heeschen, Volker. L. Weisgerber. Grundfragen der Linguistik. Mit einem Beitrag von V. Heeschen, v. Claus Heeschen, 54-69. (Urban-Tb., 156). Stuttgart.

-. 1977. Weltansicht - Reflexionen über einen Begriff W. v. Humboldts. Historiographica Linguistica VI, 2. 159-90.

Heidolph, Karl Erich [et al.]. 1981. Grundzüge einer deutschen Grammatik. Berlin.

Helbig, Gerhard. 1974. Geschichte der neueren Sprachwissenschaft. Reinbek.

Henzen, W. 1959. Rez. v. L. Weisgerber, Verschiebungen in den sprachlichen Einschätzungen von Menschen und Sachen. 1958. Beiträge zur Geschichte der deutschen Sprache und Literatur (PBB, Tübingen) 81. 203-17.

Humboldt, Wilhelm von. $1906 \mathrm{ff}$. Gesammelte Schriften, hrsg. v. A. Leitzmann. Berlin Reproduktion 1968. Berlin.

Kolb, Herbert. 1960. Der inhumane Akkusativ. Zeitschrift für deutsche Wortforschung 3.168-77.
Leuninger, Helen. 1979. Reflexionen über die Universalgrammatik. Frankfurt/M.

Motsch, Wolfgang, und Dieter Viehweger. 1981. Sprachhandlung, Satz und Text. Sprache und Pragmatik. Lunder Symposium 1980, hrsg. v. Inger Rosengren, 125-53. (Lunder germanistische Forschungen 50). Lund.

Polenz, Peter von. 1969. Der Pertinenzdativ und seine Satzbaupläne. Festschrift für Hugo Moser zum 60. Geburtstag, hrsg. v. Ulrich Engel et al., 146-71. Düsseldorf.

Růžička, Rudolf. Autonomie und Interaktion von Syntax und Semantik. Untersuchungen zur Semantik, hrsg. v. Rudolf Růžička \& Wolfgang Motsch, 15-59. (Studia Grammatica 22). Berlin, DDR.

Schoenthal, Gisela. 1976. Das Passiv in der gesprochenen deutschen Standardsprache. (Heutiges Deutsch I/7). München.

-. 1987. Kontextsemantische Analysen zum Passivgebrauch im heutigen Deutsch. Zur Mitteilungsperspektive im Passivsatz. Das Passiv im Deutschen, Nizza 1986, hrsg v. Centre de Recherche en Linguistique Germanique (Nice). (Linguistische Arbeiten 183), $161-79$. Tübingen.

Steger, Hugo. 1964. Gruppensprachen. Ein methodisches Problem der inhaltsbezogenen Sprachbetrachtung. Zeitschrift für Mundartforschung 31. $125-38$.

Szemerényi, Oswald. 1982. Richtungen der modernen Sprachwissenschaft II. Die fünfziger Jahre. Heidelberg.

Tschirch, Fritz. 1954. Weltbild, Denkform und Sprachgestalt. Grundauffassungen und Fragestellungen in der heutigen Sprachwissenschaft. (Erkenntnis und Glaube 13). Berlin.

Wandruszka, Mario. 1961. Das Passivum in den romanischen Sprachen, im Englischen und Deutschen. Der Deutschunterricht 13, 5. 40-6.

Wegener, Heide. 1985. Der Dativ im heutigen Deutsch. (Studien zur deutschen Grammatik 28). Tübingen.

Weisgerber, Leo. 1953. Vom Weltbild der deutschen Sprache. 1. Halbband, Die inhaltbezogene Grammatik. (Von den Kräften der deutschen Sprache 1). 2., erw. Aufl. Düsseldorf.

-. 1953/54. Rez. von H. Glinz, Die innere Form des Deutschen. 1952. Wirkendes Wort 4. 116-17.

-. 1957/58. Der Mensch im Akkusativ. Wirkendes Wort 8. 193-205.

-. 1962 a. Grundzüge der inhaltbezogenen Grammatik. (Von den Kräften der deutschen Sprache 1). 3., neubearb. Aufl. Düsseldorf.

-. 1962 b. Die ganzheitliche Betrachtung eines Satzbauplanes. 'Er klopfte seinem Freunde auf die Schulter'. Wirkendes Wort, Beihefte 1. Düsseldorf.

-. 1963 a. Die wirkungbezogene Sprachbetrachtung. Wirkendes Wort 13. 264-76. 
-. 1963 b. Die Welt im 'Passiv'. Die Wissenschaft von deutscher Sprache und Dichtung. Methoden, Probleme, Aufgaben. Festschrift für Friedr. Maurer, hrsg. v. S. Gutenbrunner et al., 25-59. Stuttgart.

-. 1963 c. Die vier Stufen in der Erforschung der Sprachen. (Sprache und Gemeinschaft, Grundlegung 2). Düsseldorf.
-. 1964. Vierstufige Wortbildungslehre. Muttersprache 1964, 33-43, 96.

-. 1973. Gefärbte Brillen. Linguistische Studien III. Festschrift für Paul Grebe, Teil 1. (Sprache d. Gegenwart 23), 9-23. Düsseldorf.

Jürgen Dittmann, Freiburg/Br. (Deutschland) 